\title{
A flora melitófila de uma área de dunas com vegetação de caatinga, Estado da Bahia, Nordeste do Brasil ${ }^{1}$
}

\author{
Ana Tereza Araújo Rodarte², Fabiana Oliveira da Silva ${ }^{3}$ e Blandina Felipe Viana ${ }^{3,4}$
}

Recebido em 3/04/2006. Aceito em 4/06/2007

\begin{abstract}
RESUMO - (A flora melitófila de uma área de dunas com vegetação de caatinga, Estado da Bahia, Nordeste do Brasil). As espécies melitófilas de uma área de caatinga foram caracterizadas quanto à morfologia e recursos florais, floração e abelhas visitantes. As coletas

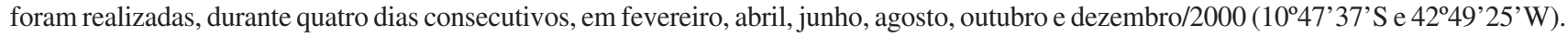
A área foi percorrida das 06:00 às 17:00 h, seguindo dois transectos paralelos (com $450 \mathrm{~m} \times 100 \mathrm{~m}$ e $550 \mathrm{~m} \times 100 \mathrm{~m}$ ) e distando $50 \mathrm{~m}$ entre si, abrangendo 10 ha. As 42 espécies melitófilas identificadas (55\% da flora local) foram visitadas por 2.924 indivíduos de 41 espécies abelhas. As famílias Caesalpiniaceae e Malpighiaceae foram as mais visitadas. As espécies predominantemente visitadas por 35 espécies de abelhas, correspondendo a 78\% do total de indivíduos foram: Byrsonima blanchetiana Miq., Copaifera coriacea Mart., Senna macranthera, Peltogyne pauciflora Benth., Senna gardneri (Benth.) H.S. Irwin \& Barneby, Serjania comata, Mouriri pusa (Gardner), Jatropha mutabilis (Pohl.) Baill., Maytenus rigida Mart. e Turnera calyptrocarpa Urb. (24\% da flora apícola). A produção de flores dessas espécies representou $95 \%$ do total. Em geral, os aspectos básicos da morfologia floral das espécies: pequenas, dispostas em inflorescências, zigomorfas, de cores alva e rosa, monoclinas e com deiscência longitudinal das anteras, não diferiram em relação à flora local, corroborando o seu caráter generalista. O predomínio de interações generalistas deve-se à abundância local de abelhas eussociais nativas (Meliponinae) e Apis mellifera. Embora a produção de flores tenha sido maior na estação chuvosa, as abelhas foram mais abundantes no período seco, indicando que o recurso não limitou a atividade das abelhas. As plantas do estrato arbustivo e herbáceo floresceram mais intensamente no período seco e chuvoso, respectivamente, gerando um grau de compartimentalização no padrão de uso dos recursos pelas abelhas. Esse padrão pode favorecer a manutenção das abelhas solitárias, de pequeno porte, incapazes de competir eficientemente com abelhas eussociais com alta capacidade de comunicação e exploração dos recursos mais abundantes como Apis mellifera. Os resultados indicaram que não apenas a composição florística, mas a estratificação e a forte sazonalidade da floração foram determinantes da composição e do padrão local de uso de recursos pelas abelhas em caatinga.
\end{abstract}

Palavras-chave: caatinga, floração, melitofilia, morfologia floral, recursos florais

\begin{abstract}
The bee flora of caatinga vegetation on sand dunes in Bahia State, Northeastern Brazil). The plant species in an area of caatinga vegetation $\left(10^{\circ} 47^{\prime} 37^{\prime} \mathrm{S}\right.$ and $\left.42^{\circ} 49^{\prime} 25^{\prime} \mathrm{W}\right)$ were surveyed to identify flower morphology, available floral resources, flowering characteristics, and visiting bee species. Collections were made for four consecutive days in February, April, June, August, and December/2000. The area was surveyed between 06:00 and 17:00 h by following two parallel transects $(450 \mathrm{~m} \times 100 \mathrm{~m}$ and $550 \mathrm{~m} \times 100 \mathrm{~m}), 50 \mathrm{~m}$ apart (total of 10 hectares). The 42 plant identified (55\% of the local flora) were visited by a total of 2924 bees belonging to 41 species. The plant families Caesalpiniaceae and Malpighiaceae were the most frequently visited. The plant species most often visited by 35 species of bees (78\% of the total number of bees surveyed) were: Byrsonima blanchetiana Miq., Copaifera coriacea Mart., Senna macranthera, Peltogyne pauciflora Benth., Senna gardneri (Benth.) H.S. Irwin \& Barneby, Serjania comata, Mouriri pusa (Gardner), Jatropha mutabilis (Pohl.) Baill., Maytenus rigida Mart. and Turnera calyptrocarpa Urb. (24\% of the total bee flora). These plant species were responsible for $95 \%$ of total flower production during the study period. Generally, the basic floral morphology of these species consisted of small, white to pink, zygomorphic flowers displayed in inflorescences, monoclinous, with longitudinal anthers dehiscence; this morphology was very similar to that of the flora, suited to generalist pollinators. The predominance of generalist interactions reflects the local abundance of stingless bees (Meliponinae) and Apis mellifera. Although flower production was greatest during the rainy season, bees were more abundant during the dry season. This indicates that bee activity was not limited by the availability of floral resources. Plants of the shrub stratatum flowered more intensely during the dry season, while herbaceous plants did so during the rainy season. This created a degree of compartmentalization of floral resources for the bees. This flowering pattern tends to favor small, solitary bees that cannot compete efficiently with eusocial bees (such as Apis mellifera) that have great capacity for communication and for exploiting the most abundant resources. The results indicate that it is not only the composition of the floral resources, but also the stratification and strong seasonal nature of flowering that influences the composition and local use by bees in caatinga vegetation.
\end{abstract}

Key words: caatinga, flowering, mellitophily, floral morphology, floral resources

\footnotetext{
1 Parte da Dissertação de Mestrado da primeira Autora

2 Universidade Federal do Rio de Janeiro, Museu Nacional, Departamento de Botânica, Quinta da Boa Vista, São Cristóvão, $20940-040$ Rio de Janeiro, RJ, Brasil

3 Universidade Federal da Bahia, Instituto de Biologia, Departamento de Zoologia, Laboratório de Biologia e Ecologia de Abelhas (LABEA), Campus Ondina, 40210-170 Salvador, BA, Brasil

4 Autor para correspondência: blande237@yahoo.com.br
} 


\section{Introdução}

O conhecimento da diversidade dos polinizadores e das suas relações com a comunidade vegetal é de fundamental importância já que a polinização é um processo chave para a conservação da biodiversidade. Dentre os polinizadores podemos encontrar agentes bióticos como insetos, pássaros e morcegos, e abióticos como água e vento (Proctor et al. 1996; Nabhan \& Buchmann 1997). As abelhas (Hymenoptera: Apoidea) reúnem aproximadamente 25.000 a 30.000 espécies, distribuídas praticamente por toda parte do mundo, sendo que as abelhas solitárias compreendem cerca de $85 \%$ da diversidade específica e são abundantes nos ecossistemas da região tropical (Michener 2000).

Cerca de $90 \%$ das angiospermas atuais são polinizadas por animais, especialmente insetos (Buchmann \& Nabhan 1996). Pelo menos 67\% das espécies de Angiospermas são polinizadas por abelhas (Faegri \& Van der Pijl 1979) que, por serem visitantes florais obrigatórios, constituem o grupo de polinizadores mais eficientes (Bawa 1990; Kevan et al. 1990; Proctor et al. 1996).

Em geral, a despeito de sua relação taxonômica, flores polinizadas por determinados visitantes compartilham um conjunto típico de características comuns, relacionadas ao tamanho, comportamento e outras características biológicas dos seus visitantes. Este conjunto de atributos é conhecido como síndrome de polinização, de modo que, por exemplo, as flores polinizadas por abelhas são ditas melitófilas (Faegri \& Van der Pijl 1979) e, muitas vezes, apresentam as seguintes características: flores com simetria zigomorfa, cores vistosas (amarelo, azul e lilás), antese diurna e odor agradável.

Os interesses antagônicos de plantas e animais forrageadores direcionaram a evolução em sentido divergente para ambos (Kearns \& Inouye 1997). Para as plantas, o pólen tem função reprodutiva, enquanto as abelhas o utilizam como alimento essencial para adultos e larvas de modo que, necessariamente, as duas funções do grão-de-pólen - reprodução e atração dos polinizadores - são mutuamente exclusivas (Westerkamp 1996). Apenas quando em excesso, o grão-de-pólen realiza as duas funções, pois, ao ser utilizado como alimento, perde a sua função reprodutiva (Barth 1985).

$\mathrm{Na}$ caatinga, embora predominem as interações generalistas, estudos realizados sobre a fauna e flora melitófila revelam a existência de um alto percentual de espécies vegetais com características melitófilas
(Freitas \& Silva 2006; Martins 1994; Aguiar et al. 1995; A.M.C. Carvalho, dados não publicados, M.S. Castro, dados não publicados). Algumas espécies são autoincompatíveis e dependem de vetores de pólen para a sua fecundação (Machado \& Lopes 2004). Contudo, a representatividade de sistemas de auto-incompatibilidade precisa ser mais investigada para permitir generalizações para a Caatinga.

Tendo em vista as lacunas existentes no conhecimento sobre flora melitófila nos ambientes de caatinga, o objetivo do presente estudo foi identificar as espécies melitófilas em uma área de dunas interiores com vegetação de caatinga e caracteriza-las quanto ao hábito, densidade, morfologia floral e floração no período estudado.

\section{Material e métodos}

Local de estudo - $\mathrm{O}$ estudo foi desenvolvido em uma área de 10 ha de dunas distando cerca de $2 \mathrm{~km}$ da margem esquerda do rio São Francisco, inserida na Área de Proteção Ambiental das Dunas e Veredas do Baixo Médio rio São Francisco, no povoado de Ibiraba, município de Barra, Bahia (1047'37"S e $42^{\circ} 49^{\prime} 25^{\prime \prime} \mathrm{W}$ ).

O clima local é Bswh, quente e semi-árido, segundo a classificação de Köppen. As chuvas se distribuem irregularmente ao longo do ano com precipitação média anual de $653,8 \mathrm{~mm}$ e a temperatura média superior a $25^{\circ} \mathrm{C}$. O solo é do tipo Neossolo Quartzarênico distrófico (Areia quartzosa distrófica), com matéria orgânica pouco espessa e sem horizonte B aparente (EMBRAPA 1999). Sobre as dunas desenvolve-se vegetação de caatinga aberta caracterizada pelo porte arbóreo arbustivo, e com baixa densidade, deixando expostas grandes porções das dunas, ocorrendo manchas compostas por cactáceas e bromeliáceas (Rocha et al. 2004).

Amostragens - As coletas foram realizadas durante o ano 2000, durante quatro dias consecutivos, a cada dois meses, abrangendo o período chuvoso (dezembro, fevereiro e abril) e seco (junho, agosto, outubro). A área foi percorrida seguindo dois transectos paralelos previamente delimitados, com auxílio de trena e bússola, e distando $50 \mathrm{~m}$ entre si; o primeiro com $450 \mathrm{~m} \times 100 \mathrm{~m}$ (50 m de cada lado) e o segundo com dimensões de $550 \mathrm{~m} \times 100 \mathrm{~m}$ (50 m de cada lado).

Coleta das abelhas - Dois coletores capturaram as abelhas em visita às flores ao longo dos transectos descritos acima, empregando o método de varredura 
(Sakagami et al. 1967). A utilização de rede entomológica com cabo longo ajustável permitiu a coleta de abelhas em flores de espécies vegetais dos estratos superior e emergente. Foram realizadas coletas bimensais, durante quatro dias consecutivos, das 6 às $17 \mathrm{~h}$, com esforço total de coleta de $264 \mathrm{~h}$. Para determinar as espécies vegetais predominantemente visitadas, calculou-se a abundância relativa (\%) dos visitantes e seus limites de confiança, pelo método de probabilidade de Kato et al. (1952 apud Laroca 1995). As abelhas estão depositadas na coleção entomológica do Laboratório de Biologia e Ecologia de Abelhas (LABEA), Instituto de Biologia, Universidade Federal da Bahia.

Caracterização da flora melitófila - As plantas foram caracterizadas quanto ao hábito em: arbórea (Arv), arbustiva (Arb), trepadeira escandente (Tesc), trepadeira (T), subarbustiva (Sarb), herbácea (Herb), hemiparasita $(\mathrm{Hp})$ e cactácea $(\mathrm{Ca})$. As exsicatas encontram-se depositadas no herbário RADAMBRASIL /IBGE (HRB), Alexandre Leal Costa (ALCB) do Instituto de Biologia da Universidade Federal da Bahia (UFBA) e da Universidade Estadual de Feira de Santana (HUEFS). A determinação das espécies foi feita por comparação com exsicatas dos herbários HRB, ALCB e HUEFS e consulta a especialistas. A classificação das famílias botânicas seguiu o sistema de Cronquist (1981).

Com base na altura, as espécies vegetais foram distribuídas nos estratos: inferior (altura $<1 \mathrm{~m}$ ); superior (altura entre 1 e $5 \mathrm{~m}$ ) e emergente (altura $>5 \mathrm{~m}$ ). A densidade das espécies do estrato superior, formado por cactáceas, arbustivas e trepadeiras lenhosas, e do estrato emergente, formado por árvores, foi medida pelo método dos quadrantes centrados (Greig-Smith 1983), marcando-se 39 pontos amostrais distantes $25 \mathrm{~m}$ entre si, ao longo de um transecto. Cada ponto amostral foi dividido em quatro quadrantes e medida a distância até o primeiro indivíduo, com uma trena. A densidade das espécies do estrato inferior (herbáceas) foi medida pelo método dos quadrantes de Braun-Blanquet (1950), com modificações. Um quadrado com dimensões de $1,0 \times 1,0 \mathrm{~m}$ era jogado aleatoriamente alternadamente para a direita e para a esquerda, a cada $25 \mathrm{~m}$ de distância, totalizando 41 pontos de amostragem. Em cada quadrado, contou-se o número de indivíduos e espécies do estrato herbáceo.

A cada amostragem registrou-se a presença/ ausência de flores e intensidade da floração das espécies visitadas por abelhas. A produção diária de flores, de cada espécie, foi estimada contando-se as flores ou inflorescências em uma amostra de 1-25 indivíduos, utilizando-se um quadrado medindo $50 \times 50 \mathrm{~cm}$ colocado aleatoriamente sobre a copa da planta. Para algumas espécies de pequeno porte, cujas dimensões da copa eram inferiores às do quadrado utilizado, contaram-se todas as flores presentes em um dado indivíduo. A estimativa da produção de flores por indivíduo foi obtida multiplicando-se o número de flores pela área total da copa do indivíduo amostrado. Para as plantas com inflorescências multiplicou-se o número médio de flores em cada inflorescência pelo número total de inflorescências na área do quadrado. A floração das espécies foi classificada como forte $(>50 \%)$, moderada $(=50 \%)$ ou fraca $(<50 \%)$, considerando a produção máxima de flores, medida para os indivíduos de cada espécie, nos seis dias de amostragem.

O número de flores estimado para a flora melitófila, apresentado neste estudo, foi comparado com a estimativa da produção total de flores apresentadas por Neves \& Viana (2002). A partir do número médio de flores por indivíduo, foi estimado o número de flores das espécies vegetais floridas na área de estudo, tomando-se os dados de densidade de cada espécie, para cada dia de coleta. Assim, o número de flores e indivíduos floridos foi obtido a partir da soma dos seis dias de estimativa.

A identificação da síndrome de melitofilia seguiu os critérios de Faegri \& Van der Pijl (1979), com base na observação dos caracteres florais em campo, com auxílio de uma lupa de bolso, ou em caso de flores muito pequenas, no laboratório, a partir de amostras de flores conservadas em álcool 70\%. As flores foram caracterizadas quanto aos seguintes parâmetros, de acordo com Dafni et al. (2005): disposição (se isoladas ou em inflorescências), coloração, forma, simetria (zigomorfa ou actinomorfa), sexualidade (monoclina ou diclina), dimensões da corola (profundidade e diâmetro) e o tipo de recurso disponível aos visitantes (néctar, pólen, óleo, resina).

Análise dos dados - O índice de Sorensen (Southwood 1980) comparou a similaridade entre as espécies melitófilas de Ibiraba (presente estudo) com áreas de Caatinga arbustiva aberta, em Casa Nova/BA (Martins 1994) e São João do Cariri/PB (Aguiar et al. 1995); Caatinga arbórea aberta, em Castro Alves/BA (A.M.C. Carvalho, dados não publicados) e Milagres/BA (A.M.C. Carvalho, dados não publicados). Este índice considera apenas dados binários (presença/ausência de espécies na amostra), através da fórmula: 
$\mathrm{CA}=[2 \mathrm{c} /(\mathrm{a}+\mathrm{b})] \times 100$; onde CA é o coeficiente de associação, c é o número de espécies comuns às duas amostras (1 e 2), a é o número de espécies da amostra 1 e b o número de espécies da amostra 2. A matriz de similaridade e o dendrograma foram feitos utilizando-se o programa Multivariate Statistical Package Version $3.12 \mathrm{~d}$

Os índices obtidos foram agrupados pelo método UPGMA, o qual classifica de forma hierárquica quantitativa, utilizando os valores de escala do coeficiente de similaridade empregado e aglomerado, acumula uma espécie ou grupo de espécies de cada vez, até finalmente a formação de um único agrupamento contendo todas as espécies. Nesse processo, o par com maior similaridade é agrupado primeiro, até a formação do agrupamento final com todas as espécies.

A existência de diferença significativa no número de visitantes em relação à produção de flores, no período seco e chuvoso, foi avaliada pelo teste t. A variação sazonal encontrada no número de espécies vegetais floridas nos estratos inferior, superior e emergente, foi testada pela ANOVA. Para todos os testes considerou-se o nível de significância de 5\% (Vieira 1991).

\section{Resultados}

$\mathrm{Na}$ área amostrada, foram identificadas 42 espécies melitófilas floridas, distribuídas em 37 gêneros e 24 famílias (Tab. 1).

A similaridade entre as áreas de caatinga estudadas por outros autores e aquela encontrada neste estudo para a flora melitófila das dunas de Ibiraba foi inferior a 30\%, sendo considerada baixa (Fig. 1).

Foram coletadas nas flores 41 espécies de abelhas (2.924 ind.) das famílias Apidae (33), Halictidae (3), Colletidae (2 sp.) e Megachilidae (3) (Tab. 2). Destas, $60 \%$ dos indivíduos visitaram as famílias Caesalpiniaceae (1.157 ind.) e Malpighiaceae (583 ind.). Apresentaram percentuais menores de abelhas visitantes as famílias Celastraceae $(3,56 \%)$, Euphorbiaceae $(4,24 \%)$, Fabaceae $(5,47 \%)$, Memecylaceae $(4,82 \%)$, Sapindaceae $(6,05 \%)$ e as demais famílias somaram $16,35 \%$ dos visitantes. No período chuvoso e no período seco foram coletados 1.392 e 1.532 indivíduos de abelhas, as quais foram mais abundantes nos meses de dezembro (665 ind.) e outubro (670 ind.) (Tab. 3). Com relação à riqueza de visitantes destacaram-se as famílias Fabaceae (24), Malpighiaceae (24), Caesalpiniaceae (22), Turneraceae (13), Sapindaceae (12). As demais 19 famílias, representando $45 \%$ do total, receberam no máximo visitas de 10 espécies de abelhas.

Não houve diferença significativa entre o número total de espécies floridas nos seis dias de amostragem, abrangendo o período seco $(n=30)$ e chuvoso $(n=26)$ $(\mathrm{F}=1,92 ; \mathrm{gl}=1)$. O menor $(\mathrm{n}=10)$ e o maior $(\mathrm{n}=21)$ número de espécies floridas ocorreu no final e início do período seco, respectivamente (Tab. 1). Cerca de $60 \%$ das espécies estavam floridas em 2 ou 3 episódios de coleta; $38 \%$ das espécies estavam floridas em pelo menos um dos meses amostrados, havendo rara sobreposição entre as espécies. Apenas Dioclea marginata estava florida em todas as amostragens, com pico em meados do período chuvoso. A maioria das espécies floresceu intensamente durante o período seco $(55 \%)$, com redução na intensidade com a proximidade do período chuvoso.

Em todos os períodos, a produção de flores das espécies visitadas por abelhas em Ibiraba foi superior a $95 \%$ da produção total estimada para a flora local, com exceção do final do período chuvoso (88\%) (Tab. 4). Copaifera coriacea (Caesalpiniaceae) e Byrsonima blanchetiana (Malpighiaceae), que floresceram nos dois períodos, corresponderam a $94 \%$ do total de flores, sendo visitadas por $74 \%$ dos indivíduos visitantes. Na estação chuvosa, a produção de flores foi superior ao produzido durante a estação seca. O pico de produção de flores ocorreu no meio do período chuvoso e no início da estação seca.

As espécies predominantemente visitadas foram Byrsonima blanchetiana, Copaifera coriacea, Senna macranthera, Peltogyne pauciflora, Senna gardneri, Serjania comata, Mouriri pusa, Jatropha mutabilis, Maytenus rigida, Turnera calyptrocarpa, as quais representaram $24 \%$ da flora melitófila e estavam distribuídas em sete famílias. A produção de flores das dez espécies vegetais mais visitadas por abelhas representou 95\% do total (Tab. 5). As espécies com maior e menor número de flores foram, respectivamente, Copaifera coriacea (3.206.888 flores) e Senna macranthera (1.299 flores).

Nas espécies vegetais predominantemente visitadas foram coletadas 35 espécies de abelhas, correspondendo a $78 \%$ do total de indivíduos. Contudo, não houve correlação $\left(r^{2}=0,189 ; p=0,209\right)$ entre $o$ número de flores produzidas e o número de indivíduos de abelhas coletados. Poucas espécies de abelhas foram coletadas em uma única espécie vegetal, ou seja, a maioria delas visitou várias plantas. As abelhas que visitaram o maior número de plantas foram: Trigona spinipes (27 sp.), Apis mellifera (20 sp.) e 
Tabela 1. Espécies melitófilas nas dunas de Ibiraba, BA, Brasil no ano de 2000: intensidade da floração (forte $\mathbf{\square}$, moderada $\square$ e fraca $\square$ ), estrato (E - emergente, S - superior e I - inferior);

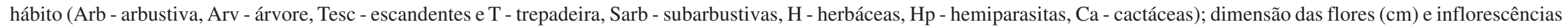
(entre parênteses); cor da corola ( A - alva, Am - amarela, C - creme, Vm - vermelha, R - rosa, V - verde, Ev - esverdeada, L - lilás, La - laranja, S - salmão, Rx - roxo); e o número de indivíduos e espécies de abelhas visitantes florais coletados.

\begin{tabular}{|c|c|c|c|c|c|c|c|c|c|c|c|c|c|c|}
\hline \multirow[t]{2}{*}{ Família/Espécie } & \multirow[t]{2}{*}{ n. } & \multirow[t]{2}{*}{ Hábito } & \multirow[t]{2}{*}{ Estrato } & \multirow{2}{*}{$\begin{array}{l}\text { Comprimento e } \\
\text { largura da corola } \\
\quad \text { (infl.) } \mathrm{cm}\end{array}$} & \multirow[t]{2}{*}{$\begin{array}{l}\text { Cor da } \\
\text { corola }\end{array}$} & \multirow{2}{*}{$\begin{array}{l}\text { Ind. } \\
\text { floridos/ } \\
\text { coleta }\end{array}$} & \multicolumn{6}{|c|}{$\begin{array}{l}\text { Mês/período seco (S) } \\
\text { e chuvoso (C)/floração }\end{array}$} & \multicolumn{2}{|c|}{$\begin{array}{c}\text { Abelhas } \\
\text { visitantes }\end{array}$} \\
\hline & & & & & & & $\mathrm{F}-\mathrm{C}$ & $\mathrm{A}-\mathrm{C}$ & $\mathrm{J}-\mathrm{S}$ & A-S & $\mathrm{O}-\mathrm{S}$ & $\mathrm{D}-\mathrm{C}$ & sp. & ind. \\
\hline \multicolumn{15}{|l|}{ ASTERACEAE } \\
\hline Vernonia lilacina Mart. ex DC. & 1 & Arb & $\mathrm{S}$ & $0,8 \times 0,1(0,9 \times 1)$ & $\mathrm{L}$ & 1 & & $\mathbf{\square}$ & & & & & 4 & 75 \\
\hline \multicolumn{15}{|l|}{ CANTHACEAE } \\
\hline Harpochilus neesianum Mart. & 2 & $\mathrm{H}$ & $\mathrm{S}$ & $9 \times 5(40 \times 20)$ & $\mathrm{V}$ & $9-13$ & & घ & $\square$ & & & & 3 & 35 \\
\hline \multicolumn{15}{|l|}{ BIGNONIACEAE } \\
\hline Proterantha sp. n & 3 & $\mathrm{~T}$ & $\mathrm{~S}$ & $3,1 \times 3,3(110 \times 40)$ & $\mathrm{R}$ & 3 & & & & $\mathbf{\square}$ & & & 10 & 51 \\
\hline \multicolumn{15}{|l|}{ BOMBACACEAE } \\
\hline Bombacopsis retusa (Mart. Ex Zucc.) A. Robyns & 4 & Arv & $\mathrm{E}$ & $9 \times 6$ & A & 7 & & & & & 曰 & & 5 & 6 \\
\hline \multicolumn{15}{|l|}{ CACTACEAE } \\
\hline Tacinga inamoena K. Sch. & 5 & $\mathrm{H}$ & I & $1,9 \times 3,5$ & $\mathrm{La}$ & $4-30$ & & $\square$ & $\square$ & $\mathbf{\square}$ & & & 2 & 25 \\
\hline Pereskia bahiensis W.R. Guerke & 6 & $\mathrm{Ca}$ & $\mathrm{S}$ & $*$ & A & 1 & & & घ & & & & 1 & 1 \\
\hline Pilosocereus gounellei (F.A.C. Weber) Byles \& G.D. Rowley & 7 & $\mathrm{Ca}$ & $\mathrm{S}$ & * & A & $1-7$ & & & $\mathbf{\square}$ & $\square$ & & & 1 & 3 \\
\hline \multicolumn{15}{|l|}{ CAESALPINIACEAE } \\
\hline Copaifera coriacea Mart. & 8 & Arb & $\mathrm{S}$ & $0,7 \times 1,1(30 \times 12)$ & A & $2-21$ & $\mathbf{\square}$ & घ & घ & & $\square$ & $\mathbf{\square}$ & 8 & 480 \\
\hline Hymenaea eriogyne Benth & 9 & Arb & $\mathrm{S}$ & $4,1 \times 3(23 \times 15)$ & $\mathrm{C}$ & 3 & & & & & $\mathbf{\square}$ & & 2 & 16 \\
\hline Peltogyne pauciflora Benth. & 10 & Arb & $\mathrm{S}$ & $0,7 \times 1(3,5 \times 2,2)$ & A & 4 & & & & & $\mathbf{\square}$ & & 8 & 200 \\
\hline Chamaecrista flexuosa L. var. flexиosa & 11 & Sarb & I & $1,8 \times 3,8$ & $\mathrm{Am}$ & $4-8$ & $\square$ & & & & & $\mathbf{\square}$ & 1 & 1 \\
\hline $\begin{array}{l}\text { Senna macranthera var. pudibunda (Mart. ex Benth.) } \\
\text { H.S. Irwin \& Barneby }\end{array}$ & 13 & Arb & $\mathrm{S}$ & $1,5 \times 5,1(12,2 \times 3,1)$ & Am & $5-7$ & & & 曰 & $\square$ & & & 13 & 179 \\
\hline S. gardneri (Benth.) H.S. Irwin \& Barneby & 14 & Arb & $\mathrm{S}$ & $1,8 \times 3,4(5,5 \times 3,1)$ & Am & $02-25$ & & घ & घ & $\square$ & & & 14 & 230 \\
\hline \multicolumn{15}{|l|}{ CELASTRACEAE } \\
\hline Maytenus rigida Mart. & 15 & Arb & $\mathrm{S}$ & $0,2 \times 0,5(3,9 \times 2,8)$ & $\mathrm{V}$ & $11-30$ & & $\square$ & घ & $\mathbf{a}$ & & & 6 & 104 \\
\hline \multicolumn{15}{|l|}{ EUPHORBIACEAE } \\
\hline Jatropha mutabilis (Pohl.) Baill. & 16 & Arb & $\mathrm{S}$ & $(4,5 \times 6,9)$ & $\mathrm{Vm}$ & $09-38$ & $\mathbf{\square}$ & & & & $\mathbf{\square}$ & $\square$ & 8 & 124 \\
\hline \multicolumn{15}{|l|}{ FABACEAE } \\
\hline Dioclea marginata Benth. & 17 & $\mathrm{~T}$ & $\mathrm{~S}$ & $2,8 \times 4(49 \times 5)$ & $\mathrm{Rx}$ & $02-07$ & $\mathbf{\square}$ & $\square$ & $\square$ & $\square$ & $\square$ & 口 & 12 & 67 \\
\hline Rynchosia sp. & 18 & Arb & $\mathrm{S}$ & $0,3 \times 0,3(9,0 \times 0,5)$ & $\mathrm{Am}$ & $3-5$ & & & - & $\square$ & & & 1 & 3 \\
\hline Aeschynomene sp. & 22. & $\mathrm{~T}$ & $\mathrm{~S}$ & $(2,5 \times 1,2)$ & Am & $3-5$ & & & $\mathbf{\square}$ & a & $\square$ & & 15 & 79 \\
\hline A. martii Benth & 19 & $\mathrm{~T}$ & $\mathrm{~S}$ & $(2,5 \times 1,2)$ & $\mathrm{Am}$ & 1 & $\mathbf{\square}$ & & & & & & 1 & 1 \\
\hline Chaetocalyx scandens (L.) Urb. & 20 & $\mathrm{~T}$ & $\mathrm{~S}$ & $*$ & & 1 & $\mathbf{\square}$ & & & & & & 5 & 7 \\
\hline Galactia remansoana Harms. & 21 & Tesc & I & $1,5 \times 2(4,0 \times 2,7)$ & $\mathrm{L}$ & $3-12$ & $\square$ & घ & $\square$ & $\square$ & & $\square$ & 3 & 3 \\
\hline \multicolumn{15}{|l|}{ LORANTHACEAE } \\
\hline Struthanthus syringifolius Mart. & 23 & $\mathrm{Hp}$ & $\mathrm{S}$ & $*$ & Am & $1-3$ & & & & & & $\boldsymbol{\square}$ & 1 & 4 \\
\hline
\end{tabular}




\begin{tabular}{|c|c|c|c|c|c|c|c|c|c|c|c|c|c|c|}
\hline \multirow[t]{2}{*}{ Família/Espécie } & \multirow[t]{2}{*}{ n. } & \multirow[t]{2}{*}{ Hábito } & \multirow[t]{2}{*}{ Estrato } & \multirow{2}{*}{$\begin{array}{l}\text { Comprimento e } \\
\text { largura da corola } \\
\quad \text { (infl.) } \mathrm{cm}\end{array}$} & \multirow[t]{2}{*}{$\begin{array}{l}\text { Cor da } \\
\text { corola }\end{array}$} & \multirow{2}{*}{$\begin{array}{l}\text { Ind. } \\
\text { floridos/ } \\
\text { coleta }\end{array}$} & \multicolumn{6}{|c|}{$\begin{array}{l}\text { Mês/período seco }(\mathrm{S}) \\
\text { e chuvoso }(\mathrm{C}) / \text { floração }\end{array}$} & \multicolumn{2}{|c|}{$\begin{array}{c}\text { Abelhas } \\
\text { visitantes }\end{array}$} \\
\hline & & & & & & & F-C & $\mathrm{A}-\mathrm{C}$ & $\mathrm{J}-\mathrm{S}$ & A-S & $\mathrm{O}-\mathrm{S}$ & $\mathrm{D}-\mathrm{C}$ & sp. & ind. \\
\hline \multicolumn{15}{|l|}{ MALPIGHIACEAE } \\
\hline Heteropterys caducibracteata W.R. Anderson & 24 & $\mathrm{~T}$ & $\mathrm{~S}$ & $0,5 \times 1,2(5,0 \times 1,7)$ & Rs & 4 & $\mathbf{\square}$ & & & & & & 4 & 5 \\
\hline Byrsonima blanchetiana Miq. & 25 & Arb & $S$ & $0,7 \times 1,5(3,1 \times 2,4)$ & Rs & $4-32$ & $\square$ & $\square$ & & & $\mathbf{\square}$ & $\square$ & 24 & 578 \\
\hline \multicolumn{15}{|l|}{ MALVACEAE } \\
\hline Herissantia crispa (L.) Brizicky & 26 & Sarb & $\mathrm{I}$ & $*$ & Am & $1-19$ & घ & $\square$ & & & & $\mathbf{\square}$ & 4 & 7 \\
\hline Sida galheirensis Ulbr. & 27 & Sarb & I & $*$ & Am & 1 & & & $\mathbf{\square}$ & & & & 1 & 3 \\
\hline \multicolumn{15}{|l|}{ MIMOSACEAE } \\
\hline Mimosa invisa Mart. ex Colla & 28 & $\mathrm{~T}$ & $\mathrm{~S}$ & $0,3 \times 0,1(1,3 \times 2,2)$ & A & 1 & & $\mathbf{\square}$ & & & & & 2 & 2 \\
\hline M. xiquexiquensis Barneby & 29 & Sarb & $\mathrm{I}$ & $0,2 \times 0,1(6,0 \times 1,9)$ & $\mathrm{L}$ & $16-90$ & $\mathbf{\square}$ & $\square$ & & & & $\mathbf{\square}$ & 6 & 76 \\
\hline \multicolumn{15}{|l|}{ MEMECYLACEAE } \\
\hline Mouriri pusa Gardner & 30 & Arv & $\mathrm{E}$ & $0,6 \times 2,7$ & A & $1-3$ & & & & $\mathbf{\square}$ & $\square$ & & 6 & 141 \\
\hline \multicolumn{15}{|l|}{ MOLLUGINACEAE } \\
\hline Glischrothamnus ulei Pilg. & 31 & $\mathrm{H}$ & I & $*$ & $\mathrm{Ev}$ & $2-13$ & $\mathbf{\square}$ & & & & & $\square$ & 2 & 12 \\
\hline \multicolumn{15}{|l|}{ MYRTACEAE } \\
\hline Eugenia cf. dictyophleba Berg. & 32 & Arb & $\mathrm{S}$ & $1,7 \times 2,6$ & A & 5 & & & & & $\mathbf{\square}$ & & 2 & 28 \\
\hline \multicolumn{15}{|l|}{ OLACACEAE } \\
\hline Ximenia americana $\mathrm{L}$. var. americana & 33 & Arb & $\mathrm{S}$ & $0,8 \times 1,2$ & $\mathrm{Ev}$ & 2 & & & & $\mathbf{\square}$ & & & 2 & 19 \\
\hline \multicolumn{15}{|l|}{ POLYGALACEAE } \\
\hline Polygala pseudohebeclada Chodat. & 34 & $\mathrm{H}$ & I & $0,9 \times 1,3$ & $\mathrm{Rx}$ & $6-11$ & $\mathbf{\square}$ & $\square$ & & & & घ & 3 & 3 \\
\hline Alibertia humilis K. Schum. & 35 & Arb & $\mathrm{S}$ & $0,8 \times 0,4$ & A & $2-5$ & & & $\mathbf{\square}$ & $\square$ & & & 1 & 3 \\
\hline \multicolumn{15}{|l|}{ SAPINDACEAE } \\
\hline Serjania comata Radlk. & 36 & $\mathrm{~T}$ & $\mathrm{~S}$ & $1,0 \times 1,5(3,8 \times 2,6)$ & A & $3-10$ & & & $\mathbf{\square}$ & $\mathbf{\square}$ & $\square$ & & 12 & 177 \\
\hline \multicolumn{15}{|l|}{ SIMAROUBACEAE } \\
\hline Simaba ferruginea A. St.-Hil. & 37 & Arb & $\mathrm{S}$ & $1,2 \times 2,3(5,6 \times 22,8)$ & A & 4 & & & & $\mathbf{\square}$ & & & 6 & 12 \\
\hline \multicolumn{15}{|l|}{ TURNERACEAE } \\
\hline Turnera calyptrecarpa Urb. & 38 & Sarb & $\mathrm{I}$ & $1,0 \times 1,1(*)$ & Am & $12-2.629$ & $\mathbf{\square}$ & घ & $\square$ & & & & 11 & 84 \\
\hline Turnera sp. 1 & 39 & Sarb & I & $1,3 \times 2,6$ & $\mathrm{Am}$ & $1-4$ & $\square$ & $\square$ & $\mathbf{\square}$ & & & & 4 & 13 \\
\hline \multicolumn{15}{|l|}{ TURNERACEAE } \\
\hline $\begin{array}{l}\text { Piriqueta duarteana (A. St.-Hil., A. Juss. \& Cambess) Urb. } \\
\text { VERBENACEAE }\end{array}$ & 40 & Sarb & I & $1,3 \times 2,6$ & $\mathrm{~S}$ & $2-12$ & घ & $\square$ & $\mathbf{\square}$ & $\square$ & & घ & 2 & 4 \\
\hline Lantana caatinguensis Mold. & 41 & $\mathrm{~T}$ & $\mathrm{~S}$ & $0,1 \times 0,6(2,7 \times 1,5)$ & Rs & $1-2$ & $\mathbf{\square}$ & $\square$ & $\square$ & $\mathbf{\square}$ & & & 3 & 7 \\
\hline \multicolumn{15}{|l|}{ VITACEAE } \\
\hline \multirow[t]{2}{*}{ Cissus coccinea Mart. ex Planch. } & 42 & Tesc & I & $*$ & $\mathrm{Ev}$ & 03 & & & & & & $\square$ & 1 & 5 \\
\hline & & & & & & & & & & & & & 41 & 2.924 \\
\hline
\end{tabular}

* Plantas cujas dimensões das flores não foram medidas. 


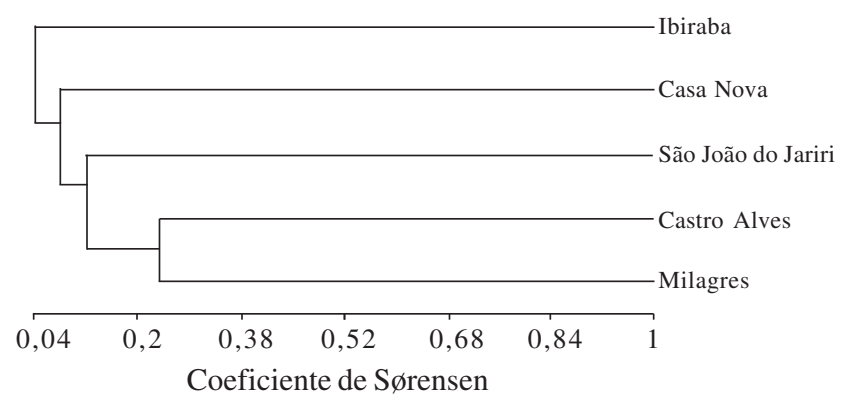

Figura 1. Similaridade entre os agrupamentos das espécies melitófilas em áreas de caatinga nas localidades de Castro Alves (A.M.C. Carvalho, dados não publicados), Milagres (M.S. Castro, dados não publicados), São João do Cariri (Aguiar et al. 1995), Casa Nova (Martins 1994).
Frieseomellita silvetrii languida (17 sp.).

Nas espécies predominantemente visitadas, a maioria das flores está disposta em inflorescências (80\%), com predomínio de flores zigomorfas (70\%) em relação às actinomorfas (30\%). Quanto à cor, a corola pode ser alva (40\%), amarela (30\%), verde $(10 \%)$, rosa $(10 \%)$ e vermelha $(10 \%)$ (Tab. 5). O diâmetro da corola variou de 0,1 a $2,0 \mathrm{~cm}$, em $83 \%$ das espécies. Estas foram visitadas por $70 \%$ das abelhas, enquanto as flores grandes, com diâmetro de 4,0 a $6,0 \mathrm{~cm}$, encontradas em $17 \%$ das espécies vegetais, foram visitadas por $10 \%$ do total de abelhas. Quanto à sexualidade, as flores são monóclinas (90\%)

Tabela 2. As abelhas visitantes da flora melitófila, de acordo com o estrato, durante os meses amostrados no ano 2000, nas dunas de Ibiraba, BA, Brasil. Código da planta conforme a Tab. 1.

\begin{tabular}{|c|c|c|c|c|}
\hline \multirow[t]{2}{*}{ Espécies de abelhas } & \multicolumn{3}{|c|}{ Plantas visitadas } & \multirow[t]{2}{*}{ N. de indivíduos } \\
\hline & Superior & Inferior & Emergente & \\
\hline \multicolumn{5}{|l|}{ APIDAE } \\
\hline Apis mellifera (Linnaeus, 1758) & $\begin{array}{c}36,23,01,33,10, \\
16,32,35,22,25,08, \\
17,15,03,13,14\end{array}$ & $27,38,29$ & 04 & 864 \\
\hline Centris sp. & 25 & & & 01 \\
\hline C. (Centris) aenea (Lepeletier, 1841) & $22,25,20,24,15,14,36$ & $12,38,39$ & & 73 \\
\hline C. (Centris) caxiensis (Ducke, 1907) & $22,25,20,41,15,10,14,36$ & $12,21,34,38,39$ & & 190 \\
\hline C. (Centris) flavifrons (Fabricius, 1775) & $25,17,36$ & & & 32 \\
\hline C. (Centris) poecila (Lepeletier, 1841) & 25 & & & 02 \\
\hline C. (Heterocentris) analis (Fabricius, 1804) & 25,24 & & & 02 \\
\hline C. (Paremisia) fuscata (Lepeletier, 1841) & $25,17,03,14$ & 12 & & 14 \\
\hline C. (Paremisia) pulchra sp. n. & 25 & 29,38 & & 04 \\
\hline C. (Melanocentris) obsoleta (Lepeletier, 1841) & $25,17,24$ & & & 11 \\
\hline C. (Ptilotopus) maranhensis (Ducke, 1910) & $25,17,28,14$ & 12 & & 25 \\
\hline C. (Ptilotopus) sponsa $($ Smith, 1854$)$ & $17,28,14$ & 12 & & 27 \\
\hline C. (Hemisiella) tarsata (Smith, 1874) & 25,20 & & & 07 \\
\hline C. (Hemisiella) trigonoides (Lepeletier, 1841) & 24,03 & 11 & & 15 \\
\hline C. (Hemisiella) vittata (Lepeletier, 1841) & 25,17 & & & 02 \\
\hline C. (Paracentris) xanthomelaena (Moure \& Castrc & ro, 2001) & 12 & & 02 \\
\hline Ceratina (Crewella) cfr. aspera (Schrottky, 1902 & 16,13 & $26,34,38$ & & 06 \\
\hline Epicharis (Epicharis) bicolor (Smith, 1854) & 25 & & & 01 \\
\hline Exomalopsis (E.) analis (Spinola, 1853) & 13 & & & 03 \\
\hline Euglossa sp. & 41 & & & 01 \\
\hline Eulaema (Apeulaema) nigrita (Lepeletier, 1841) & $03,14,01$ & 12,38 & & 27 \\
\hline Frieseomellita doederleini (Friese, 1900) & 25,37 & & 30 & 20 \\
\hline Frieseomellita (silvetrii) languida (Moure, 1989) & $\begin{array}{l}22,25,20,08,17 \\
02,09,15,06,03 \\
14,13,37,33\end{array}$ & 31,34 & 30 & 316 \\
\hline Larochantideo sp. & 22,42 & & & 15 \\
\hline Melipona (Eumelipona) asilvai (Moure, 1971) & $22,25,08,14,36,01$ & 29,38 & & 70 \\
\hline Melipona (Melipona) mandacaia (Smith, 1863) 2 & $25,08,17,32,16,10,13,36$ & 11,29 & & 61 \\
\hline Mesoplia sp. & & 38 & & 01 \\
\hline Plebeia sp. & $\begin{array}{c}22,25,08,02,16,13 \\
36,37,33\end{array}$ & $05,21,26,39$ & 04,30 & 43 \\
\hline Trigonisca sp. & $22,25,08,16,10,13,37$ & 26,38 & 30 & 158 \\
\hline Trigona spinipes (Fabricius, 1793) & $\begin{array}{c}22,25,08,17,02,09 \\
16,41,15,10,06,07,18 \\
14,13,36,37,33,36\end{array}$ & $05,12,21,29,39,38$ & 04,30 & 615 \\
\hline
\end{tabular}


Tabela 2 (continuação)

\begin{tabular}{|c|c|c|c|c|}
\hline \multirow[t]{2}{*}{ Espécies de abelhas } & \multicolumn{3}{|c|}{ Plantas visitadas } & \multirow[t]{2}{*}{ N. de indivíduos } \\
\hline & Superior & Inferior & Emergente & \\
\hline $\begin{array}{l}\text { Xylocopa }(\text { Neoxylocopa }) \text { grisescens } \\
\quad \text { (Lepeletier, 1841) }\end{array}$ & $19,08,17,16,10,03,14,13$ & 12 & 04 & 54 \\
\hline X. (Neoxylocopa) frontalis (Oliver, 1789) & 17,13 & 12 & & 06 \\
\hline X. (Schonnherria) subcyanea (Pérez, 1910) & $22,03,13,36$ & $12,40,38$ & & 11 \\
\hline \multicolumn{5}{|l|}{ COLLETIDAE } \\
\hline Perditomorpha sp. & & 26 & & 04 \\
\hline Ptiloglossa sp. & & & 04 & 01 \\
\hline \multicolumn{5}{|l|}{ HALICTIDAE } \\
\hline Algochloropsis callichroa (Cockerell, 1900) & $25,15,03,13,14,36,37$ & & 30 & 123 \\
\hline Halictus (Seladonia) hesperus (Smith, 1862) & 25,13 & & & 40 \\
\hline Pseudaugochlora pandora (Smith, 1853) & $22,16,03,14,13,36$ & 12,29 & & \\
\hline \multicolumn{5}{|l|}{ MEGACHILIDADE } \\
\hline Megachile (Pseudocentrum) sp. & 22 & & & 07 \\
\hline Megachile (Sayapis) cfr. dentipes (Vachal, 1909) & 22,01 & 21 & & 14 \\
\hline Epanthidium tigrinum (Schrottky, 1905) & 22,20 & & & 20 \\
\hline Total & & & & 2.888 \\
\hline
\end{tabular}

Tabela 3. Abundância de abelhas coletadas nas espécies vegetais melitófilas, durante o período seco e chuvoso nas dunas de Ibiraba, BA, Brasil.

\begin{tabular}{|c|c|c|c|c|c|}
\hline \multirow{2}{*}{$\begin{array}{l}\text { Estação } \\
\text { Período chuvoso }\end{array}$} & \multicolumn{4}{|c|}{ Datas/meses de coleta } & \multirow{2}{*}{$\begin{array}{c}\text { N. de } \\
\text { indivíduos }\end{array}$} \\
\hline & $1 / \mathrm{fev}$ & $2 / \mathrm{fev}$ & $3 / \mathrm{fev}$ & $4 / \mathrm{fev}$ & \\
\hline & 14 & 78 & 91 & 96 & 279 \\
\hline & $11 / a b r$ & $12 / a b r$ & $13 / a b r$ & $14 / a b r$ & \\
\hline & 130 & 81 & 88 & 150 & 448 \\
\hline & $27 / \mathrm{dez}$ & $28 / \mathrm{dez}$ & $29 / \mathrm{dez}$ & $30 / \mathrm{dez}$ & \\
\hline & 261 & 130 & 112 & 162 & 665 \\
\hline \multirow[t]{6}{*}{ Período seco } & 2/jun & 3/jun & 4/jun & 5/jun & \\
\hline & 163 & 70 & 122 & 106 & 461 \\
\hline & 4/ago & 5/ago & 6/ago & 7/ago & \\
\hline & 104 & 105 & 121 & 72 & 401 \\
\hline & $25 /$ out & 26/out & $27 /$ out & 28/out & \\
\hline & 112 & 169 & 153 & 236 & 670 \\
\hline
\end{tabular}

Tabela 4. Número estimado de flores disponíveis no campo (Neves \& Viana 2002) e de flores melitófilas em seis dias de observação, no período chuvoso e seco, nas dunas de Ibiraba, BA, Brasil.

\begin{tabular}{llrrr}
\hline \multirow{2}{*}{ Período } & & \multicolumn{3}{c}{ Número de flores disponíveis } \\
\cline { 3 - 5 } & & Toda flora & Flora melitófila & $\%$ \\
\hline \multirow{2}{*}{ Chuvoso } & Dezembro & 520.591 & 503498 & 96,7 \\
& Fevereiro & 2.929 .659 & 2920505 & 99,7 \\
\multirow{4}{*}{ Seco } & Abril & 180.546 & 159282 & 88,2 \\
& Junho & 1.022 .483 & 1015576 & 99,8 \\
& Agosto & 162.969 & 162107 & 99,3 \\
\multirow{2}{*}{ Total } & Outubro & 129.891 & 129633 & 99,8 \\
& & 4.946 .139 & 4890601 & $99 \%$ \\
\hline
\end{tabular}

ou díclinas (10\%). A deiscência pode ser longitudinal $(70 \%)$ ou poricida $(30 \%)$. Os principais recursos presentes nas flores dessas espécies foram néctarpólen (40\%) e néctar-pólen-resina (30\%). Nas demais, os recursos presentes foram pólen (10\%), resina-pólen (10\%), pólen-óleo (10\%) (Tab. 5).

A maioria das espécies, especialmente entre aquelas do estrato superior e emergente (e.g., Mouriri pusa, Senna gardneri, S. macranthera. Copaifera coriacea e Peltogyne pauciflora (Caesalpiniaceae), Serjania comata (Sapindaceae) e Maytenus rigida (Celastraceae), disponibilizou pólen e néctar aos visitantes (Tab. 6).

As duas espécies do estrato emergente floresceram no período seco, enquanto que algumas espécies do estrato superior e inferior floresceram nos dois períodos. No período chuvoso, oito espécies floresceram no estrato inferior (subarbustiva e herbáceas) e, no período seco, 15 espécies floresceram no estrato superior (arbustivas, cactáceas e trepadeiras), havendo diferença significativa $(\mathrm{F}=66,06$; $\mathrm{gl}=2 ; \mathrm{p}=5 \%)$ entre os dois estratos conforme o período do ano (Tab. 6). Nas espécies vegetais associadas ao estrato superior registrou-se maior número e diversidade de abelhas, especialmente nas arbustivas e trepadeiras, como Byrsonima blanchetiana, Copaifera coriacea e Senna macranthera. No estrato inferior, as espécies de hábito subarbustivo receberam o maior número de visitantes (242 ind.), sendo que Turnera calyptrocarpa recebeu 84 ind. (34,71\%). Das três 
Tabela 5. Espécies predominantemente visitadas por abelhas em seis dias de amostragem, nos meses de fevereiro, abril, junho, agosto, outubro e dezembro, nas dunas de Ibiraba, BA, Brasil. Estrato (S - superior, I - inferior, E - emergente), cor da corola, recursos florais, número de indivíduos floridos e a produção de flores.

\begin{tabular}{|c|c|c|c|c|c|}
\hline Espécies vegetais & Estrato & Cor das flores & Recursos tróficos & $\begin{array}{l}\text { Número de } \\
\text { indivíduos }\end{array}$ & $\begin{array}{l}\text { Número de } \\
\text { flores }\end{array}$ \\
\hline Byrsonima blanchetiana & $\mathrm{S}$ & rosa & pólen - óleo & 59 & 96914 \\
\hline Copaifera coriacea & $\mathrm{S}$ & alva & néctar - pólen - resina & 37 & 3206888 \\
\hline Senna macranthera & $\mathrm{S}$ & amarela & nécta - pólen & 12 & 1299 \\
\hline Peltogyne pauciflora & $\mathrm{S}$ & alva & nécta - pólen - resina & 4 & 59934 \\
\hline Senna gardneri & $\mathrm{S}$ & amarelo & néctar - pólen & 36 & 2058 \\
\hline Serjania comata & $\mathrm{S}$ & alva & néctar - pólen & 19 & 20707 \\
\hline Mouriri pusa & $\mathrm{E}$ & alva & néctar - pólen & 3 & 17672 \\
\hline Jatropha mutabilis & $\mathrm{S}$ & vermelha & resina - pólen & 38 & 3485 \\
\hline Maytenus rígida & $\mathrm{S}$ & verde & néctar - pólen - resina & 30 & 1207411 \\
\hline Turnera calyptrocarpa & I & amarelo & pólen & 2 & 84891 \\
\hline Total & & & & & 4701259 \\
\hline
\end{tabular}

Tabela 6. Floração das espécies e número de indivíduos de abelhas coletados nas espécies vegetais dos estratos: emergente (árvores), superior (arbustivas, cactáceas e trepadeiras) e inferior (herbáceas, trepadeiras rastejantes e subarbustos), nas dunas de Ibiraba, BA, Brasil, durante os meses amostrados. Os valores percentuais estão representados entre parênteses.

\begin{tabular}{lccccccc}
\hline \multirow{2}{*}{ Estrato } & \multicolumn{3}{c}{ Espécies vegetais } & & \multicolumn{2}{c}{ Total } \\
\cline { 2 - 3 } \cline { 7 - 8 } & Chuvoso & Seco & Duas estações & & Plantas & Abelhas \\
\hline Emergente & - & $2(100)$ & - & & 2 & 147 \\
Superior & $5(19)$ & $15(58)$ & $6(23)$ & & 26 & 2.490 \\
Inferior & $8(57)$ & $1(7)$ & $5(36)$ & & 14 & 287 \\
Total & $13(31)$ & $18(43)$ & $11(26)$ & & 42 & 2.924 \\
\hline
\end{tabular}

espécies que compõem o estrato emergente, Pterocarpus monophyllus, Mouriri pusa e Bombacopsis retusa, apenas as duas últimas foram visitadas (Tab. 1).

\section{Discussão}

O número de espécies melitófilas encontrado nas dunas de Ibiraba foi elevado (55\% das espécies) em relação à composição florística da área amostrada, que registrou 72 espécies (A.T.A. Rodarte, dados não publicados). A representatividade em termos de diversidade específica foi inferior àquela encontrada em ecossistemas similares do semi-árido (Martins 1994; Aguiar et al. 1995; A.M.C. Carvalho, dados não publicados; M.S. Castro, dados não publicados). Apesar da maior riqueza de espécies vegetais observada por M.S. Castro (dados não publicados), na região de Milagres, BA - 186 espécies amostradas, sendo $121 \mathrm{spp}$. visitadas por abelhas - houve semelhança em termos do percentual de espécies melitófilas $(66 \%)$ em relação ao obtido neste estudo $(55 \%)$.

A baixa similaridade em nível específico entre as dunas de Ibiraba e outras localidades de caatinga (A.M.C. Carvalho, dados não publicados; Martins 1994; Aguiar et al. 1995; M.S. Castro, dados não publicados) resulta do fato das dunas de Ibiraba representarem um ambiente de ecótono, com alto grau de endemismo (Barneby 1991) e espécies com distribuição disjunta (Araújo et al. 1998). Alguns estudos sugerem que as plantas das dunas do Médio Rio São Francisco representam uma unidade fitogeográfica distinta daquelas que ocupam áreas de caatinga não arenosas (Rocha et al. 2004).

A concentração das visitas das abelhas em poucas espécies da flora melitófila corrobora um padrão comum para ambientes tropicais (Ramalho et al. 1990; [Carvalho \& Bego 1997; Viana et al. 2006). Em Ibiraba, o percentual de plantas predominantemente visitadas foi superior ao registrado por A.M.C. Carvalho (dados não publicados), em Castro Alves (7,87\%), por M.S. Castro (dados não publicados) em Milagres (11,6\%) e por Aguiar et al. (1995) em São João do Cariri (21\%).

Embora as nossas observações sobre o comportamento da floração das espécies melitófilas nas dunas de Ibiraba não sejam conclusivas, foi possível verificar tendência similar ao padrão de floração descrito em estudos sobre floração na caatinga (Aguiar et al. 1995; A.M.C. Carvalho, dados não publicados; 
M.S. Castro, dados não publicados), marcado pelo assincronismo na floração e forte sazonalidade. Em algumas espécies como Aeschynomene martii (Fabaceae), Chaetocalyx scandens (Fabaceae), Mimosa invisa (Mimosaceae), a duração e intensidade da floração mostraram clara relação com a disponibilidade das chuvas, um fato comum em ambientes sazonais da região tropical (Andrade-Lima 1981; Opler et al. 1976). O maior número de espécies floresceu no período seco, havendo um elevado percentual de espécies florescendo nos dois períodos. Um padrão inverso foi observado por Machado (1990), na Caatinga arbórea de Alagoinha, PE, onde foram observadas poucas espécies com floração nos dois períodos (5\%).

Em geral, a intensidade de floração em muitas espécies nas dunas de Ibiraba não foi dependente da densidade de indivíduos na área. Dentre as seis espécies de Euphorbiaceae registradas na área estudada, apenas J. mutabillis foi predominantemente visitada por abelhas, enquanto as várias espécies do gênero Croton, apesar da alta densidade, não o foram, talvez em decorrência do baixo número de indivíduos floridos (A.T.A. Rodarte, dados não publicados). Já em espécies de Caesalpiniaceae, a intensa visitação por abelhas deveu-se principalmente ao grande número de flores abertas em determinados meses, resultante da floração sincrônica dos indivíduos dessas espécies.

A variação na intensidade e o assincronismo da floração das espécies melitófilas dispõem recursos florais continuamente, a despeito da sazonalidade. Esse padrão reduziu a sobreposição entre os picos de floração das espécies, elevando a taxa de visitação de algumas destas. Isto implica numa ininterrupta atividade dos visitantes que, porém, alternam as fontes de recursos disponíveis. Por exemplo, Copaifera coriacea no meio do período chuvoso foi uma espéciechave para a fauna de abelhas. O papel da floração na regulação da distribuição temporal da abundância e diversidade específica de alguns grupos de abelhas, e na atração e concentração de polinizadores potenciais já foi apontado por alguns autores (Janzen 1967; Bawa 1983; Newstrom et al. 1993; 1994).

Com relação à abundância de visitantes observou-se uma tendência sazonal inversa (maior no período seco) àquela observada para a disponibilidade floral (maior no período chuvoso). Outros estudos em área de caatinga observaram maior atividade de abelhas durante o período seco (Aguiar \& Martins 1997; Aguiar 2003). Deve-se considerar a influência dos fatores climáticos, que têm ação diferente sobre a fisiologia vegetal e atividade de forrageio das abelhas. Na estação chuvosa, o alto percentual de flores (77\%) resultou da coincidência dos picos de florescimento de espécies com grande número de flores, favorecidas por temperaturas mais baixas e por precipitações mais elevadas.

A menor disponibilidade de flores no período seco não limitou a atividade das abelhas nas dunas. A floração de algumas espécies do estrato superior como Jatropha mutabilis, Peltogyne pauciflora e Byrsonima blanchetiana, visitadas preferencialmente por abelhas eussociais (Neves \& Viana 2002), determinou a maior abundância de visitantes nesse período. A alta visitação às flores desse estrato deveuse à diversidade e intensidade da floração das espécies, à disposição das flores em inflorescências, concentrando os recursos e facilitando a sua visualização pelos forrageadores além da previsibilidade dos recursos ao longo do tempo. As espécies que exploraram recursos nesse estrato podem manter populações perenes, para as quais as relações generalistas são mais vantajosas.

As espécies do estrato inferior floresceram no período chuvoso sendo muito importantes para a fauna local nesse período, seja como fonte alternativa ou exclusiva de alimento. As herbáceas desse estrato são sensíveis ao estresse hídrico resultante da falta de chuvas e desaparecem durante o período seco. Assim, os recursos das plantas desse estrato são menos previsíveis no tempo, e requerem sincronismo com a atividade de forrageio dos visitantes com atividade restrita ao período chuvoso, já que estes ocorrem durante período restrito.

Em geral, os aspectos básicos da morfologia floral das espécies mais visitadas, coincidiram com aqueles descritos (A.T.A. Rodarte, dados não publicados), com pequenas alterações nas proporções, corroborando o seu caráter generalista. A flora melitófila compartilha características que contribuem para a atratividade da fauna de abelhas, como simetria zigomorfa, predomínio da cor amarela, antese diurna e produção néctar (Faegri \& Van der Pij1 1979; Dafni et al. 2005; Proctor et al. 1996). A floração intensa e a disposição das flores em inflorescências, comum entre as espécies predominantemente visitadas, podem estar incrementando a atratividade visual à longa distância como também sugerem outros autores (Machado \& Lopes 2004; Dafni et al. 1997).

A predominância de flores alvas e amarelas nas espécies vegetais mais visitadas por abelhas, observada neste estudo foi constatada em outras localidades como 
Milagres (M.S. Castro, dados não publicados) e Casa Nova (Martins 1994). A distribuição das cores das flores, nos diferentes estratos, reflete as diferenças na composição florística e à dominância na floração das espécies que compõem cada um desses estratos. Porém, a cor não foi determinante na escolha das flores pelos visitantes, sendo a oferta de recurso o fator preponderante.

A maioria das espécies, independente do estrato, produz néctar e pólen e os insetos forragearam por esses recursos, os quais são os elementos nutritivos mais comuns (Simpson \& Neff 1981). A presença de nectários extraflorais contribuiu para a atratividade das abelhas às espécies vegetais do estrato inferior como observado em Turnera calyptrocarpa (Turneraceae). O pólen de Senna macranthera e S. gardneri, em anteras poricidas, estavam acessíveis às espécies que coletam pólen por vibração (Buchmann 1983). Espécies de Xilocopini e Centridini, que voam longas distâncias e coletam pólen por vibração (Machado \& Lopes 1998; Neves \& Viana 2002), são os principais polinizadores dessas espécies localmente (Borges et al. 2002). O elevado número de estames e as dimensões das anteras, cuja cor contrasta com as pétalas, facilita a localização pelos visitantes. Contudo, nas espécies de Senna estudadas, além das características morfoestruturais da flor, a floração desempenha importante papel para atração dos polinizadores. A separação temporal do pico de floração prolonga a oferta de recursos tróficos à fauna local de abelhas, garantindo a coexistência de ambas (Borges et al. 2002).

Os resultados elucidaram alguns aspectos descritivos da interação entre a fauna de abelhas e as espécies melitófilas visitadas nas dunas de Ibiraba, e reforçaram o papel da estrutura e composição na determinação das interações no ecossistema de dunas do médio Rio São Francisco. São necessários estudos enfocando o contexto da paisagem e a distribuição dos recursos da flora melitófila para o entendimento dessa complexidade.

Embora a forte tendência à generalização seja preponderante no sistema como um todo, a forte sazonalidade, a composição florística e o comportamento diferencial da floração, podem influenciar o estabelecimento de relações preferenciais, especialmente no estrato inferior. A estratificação da flora melitófila tendeu a compartimentalizar a fauna de forrageadores, podendo favorecer a manutenção das abelhas solitárias, de pequeno porte como as do gênero Ceratina (Apidae); Pseudaugochlora, Halictus (Halictidae) e Megachile (Megachilidae), incapazes de competir eficientemente com abelhas sociais com alta capacidade de comunicação e exploração dos recursos mais abundantes como Apis mellifera.

\section{Agradecimentos}

Ao PRODOC/UFBA/CADCT e ao CNPq, pelos auxílios financeiros dados ao projeto de pesquisa; aos professores Geraldo Carlos Pereira Pinto (IBGE), Maria Lenise S. Guedes (UFBA) e Luciano Paganucci de Queiroz (UEFS), pela identificação das espécies vegetais; à equipe do LABEA, pelo auxílio nas atividades de campo; ao INMET- IV Distrito, pelo fornecimento dos dados meteorológicos; e aos revisores dessa Revista cujas valiosas contribuições foram incorporadas à versão final desse artigo.

\section{Referências bibliográficas}

Aguiar, C.M.L. 2003. Utilização de recursos florais por abelhas (Hymenoptera: Apoidea) em uma área de Caatinga (Itatim, Bahia, Brasil). Revista Brasileira de Zoologia 20: 457-467.

Aguiar, C.M.L. \& Martins, C.F. 1997. Abundância relativa, diversidade e fenologia de abelhas (Hymenoptera, Apoidea) na caatinga, São João do Cariri, Paraíba, Brasil. Iheringia, Ser. Zool. 83: 151-163.

Aguiar, C.M.L.; Martins, C.F. \& Moura, A.C.A. 1995. Recursos florais utilizados por abelhas (Hymenoptera, Apoidea) em área de caatinga (São João do Cariri, Paraíba). Revista Nordestina de Biologia 10: 101-117.

Andrade-Lima, D. 1981. The caatingas dominium. Revista Brasileira de Botânica 4: 142-153.

Araújo, F.S.; Sampaio, E.V.S.B.; Figueiredo, M.A.; Rodal, M.J.N. \& Fernandes, A.G. 1998. Composição florística da vegetação de carrasco, Novo Oriente - CE. Revista Brasileira de Botânica 21: 15-26.

Barth, F.G. 1985. Insects and flowers: the biology of a partnership. New Jersey, Princeton University.

Barneby, R. 1991. Sensitivae Censitae, a description of the genus Mimosa L. (Mimosaceae) in the New World. Memoirs of The New York Botanical Garden 65: 1-835.

Bawa, K.S. 1983. Patterns of flowering in tropical plants Pp. 394-410. In: C.E. Jones \& R.K. Little (eds.). Handbook of Experimental Pollination Ecology. New York, Von Nostrand Reinhold.

Bawa, K.S. 1990. Plant-pollinator interactions in tropical rain forests. Annual Review of Ecology and Systematics 21: 399-422.

Braun-Blanquet, J. 1950. Sociologia vegetal: estúdio de las comunidades vegetais. Buenos Aires, Acme.

Buchmann, S.L. 1983. Buzz pollination in angiosperms Pp. 73-113. In: C.E. Jones \& R.J. Little (eds.). Handbook of experimental pollination biology. New York, Scientific and Academic Editions. 
Buchmann, S.L. \& Nabhan, G.P. 1996. The forgotten pollinators. Washington D.C., Island Press.

Borges, M.S.; Viana, B.F. \& Neves, E.L. 2002. Aspectos da estratégia reprodutiva de duas espécies co-ocorrentes de Senna (K. Bauhin) P. Miller (Caesalpiniaceae) nas dunas do Médio Rio São Francisco, Bahia. Sitientibus 2: $49-54$.

Carvalho, A.M.C. \& Bego, L.R. 1997. Exploitation of available resources by bee fauna (ApoideaHymenoptera) in the Reserva Ecológica do Panda, Uberlândia, State of Minas Gerais, Brazil. Revista Brasileira de Entomologia 41: 101-107.

Cronquist, A. 1981. An integrated system classification of flowering plants. New York, Columbia University.

Dafni, A.; Kevan, P.G. \& Husband, B.C. (eds.). 2005. Practical pollination biology. Cambridge, Cambridge University Press.

Dafni, A.; Lehrer, M. \& Kevan, P.G. 1997. Spatial flower parameters and insect spatial vision. Biological Review 72: 239-282.

EMBRAPA. 1999. Sistema Brasileiro de Classificação do solo. Rio de Janeiro, Centro Nacional de Pesquisas do Solo.

Faegri, K. \& Van der Pijl, L. 1979. The principles of pollination ecology. $3^{\text {th }}$ ed. London, Pergamon.

Freitas, B.M. \& Silva, E.M.S. 2006. Potencial apícola da vegetação do semi-árido brasileiro. Pp. 19-32. In: A.M. Giulietti \& L.P. Queiroz (orgs.). Apium plantae. Recife, Instituto do Milênio do Semi-Árido.

Greig-Smith, P. 1983. Quantitative plant ecology. Oxford, Blackwell.

Janzen, D.H. 1967. Syncronization of sexual reproduction of trees within the dry season in central America. Evolution 21: 620-537.

Kearns, C.A. \& Inouye, D.W. 1997. Pollinators, flowering plants, and conservation biology. Bio-Science 47: 297-307.

Kevan, P.G.; Clark, E.A. \& Thomas, V.G. 1990. Insect pollinators and sustainable agriculture. American Journal of Alternative Agriculture 5: 13-22.

Laroca, S. 1995. Ecologia: princípios e métodos. Petrópolis, Vozes.

Machado, I.C.S. \& Lopes, A.V. 2004. Floral traits and pollination systems in the Caatinga, a Brazilian tropical dry forest. Annals of Botany 94: 365-376.

Machado, I.C.S. \& Lopes A.V. 1998. A polinização biótica e seus mecanismos na Reserva Ecológica de Dois Irmãos. Pp. 173-195. In: I.C.S. Machado; A.V. Lopes \& K.C. Porto (eds.). Reserva ecológica de Dois Irmãos: estudos em um remanescente de mata atlântica em área urbana (Recife-Pernambuco-Brasil). Recife, Ed. Universitária da UFPE.

Martins, C.F. 1994. Comunidade de abelhas (Hymenoptera, Apoidea) da caatinga e do cerrado com elementos de campo rupestre do estado da Bahia, Brasil. Revista Nordestina de Biologia 9: 225-257.

Michener, C.D. 2000. The bees of the world. Baltimore, Johns Hopkins University.
Nabhan, G.P. \& Buchmann, S.L. 1997. Services provided by pollinators. Pp. 133-50. In: G.C. Daily (ed.). Nature's service: Societal dependence on natural ecosystems. Washington, D.C., Island.

Neves, E.L. \& Viana, B.F. 2002. As abelhas eussociais (Hymenoptera, Apidae) visitantes florais em um ecossistema de dunas continentais no médio Rio São Francisco, Bahia, Brasil. Revista Brasileira de Entomologia 46: 573-580.

Newstrom, L.E.; Frankie, G.W.; Baker, H.G. \& Colwel, R.K. 1993. Diversity of long-term flowering patterns. Pp. 142-160. In: L.A. McDade; K.S. Bawa; G.S. Hartshorn \& H.A. Hespenheide (eds.). La Selva: Ecology and natural history of a lowland tropical rainforest. Chicago, University of Chicago.

Newstrom, L.E.; Frankie, G.W. \& Baker, H.G. 1994. A new classification for plant phenology based on flowering patterns in lowland tropical rain forest trees at La Selva, Costa Rica. Biotropica 26: 141-159.

Opler, P.A.; Frankie, G.W. \& Baker, H.G. 1976. Rainfall as a factor in the release, timing and synchronization of anthesis by tropical trees and schrubs. Journal of Biogeography 3: 36-231.

Proctor, T.; Yeo, P. \& Lack, A. 1996. The natural history of Pollination. London, Harper Collins.

Ramalho, M.; Kleinert-Giovannini, A. \& Imperatriz Fonseca, V.L. 1990. Important bee plants for stingless bees (Melípona and Trigonini) and Africanized honey bees (Apis mellifera) in neotropical habitats: a review. Apidologie 21: 469-488.

Rocha, P.L.B.; Queiroz, L.P. \& Pirani, J.R. 2004. Plant species and habitat structure in a sand dune field in the Brazilian Caatinga: a homogeneous habitat harbouring and endemic biota. Revista Brasileira de Botânica 27: 739-755.

Sakagami, S.F.; Laroca, S. \& Moure, J.S. 1967. Wild bee biocenotics in São José dos Pinhais (Pr), South Brazil. Preliminary report. Journal Faculty Hokkaido University 19: 190-250.

Simpson, B.B. \& Neff, J.L. 1981. Floral rewards: alternatives to pollen and nectar. Annals of the Missouri Botanical Gardens 68: 301-322.

Southwood, T.R.E. 1980. Ecological Methods - with particular reference to the study of insect populations. London, Chapman and Hall.

Viana, B.F.; Silva, F.O. \& Kleinert, A.M.P. 2006. A flora apícola de uma área restrita de dunas litorâneas, Abaeté, Salvador, Bahia. Revista Brasileira de Botânica 29: 13-25.

Vieira, S. 1991. Introdução a bioestatística. $2^{\text {a }}$ ed. Rio de Janeiro, Campus.

Vogel, S. 1978. Evolutionary shifts from reward to deception in pollen flowers. Pp. 89-96. In: A.J. Richards (ed.). The pollination of flowers by insects. New York, Academic Press.

Westerkamp, C. 1996. Pollen in bee-flower relations: some considerations on melittophily. Botanical Acta 109: 325-332.

Versão eletrônica do artigo em www.scielo.br/abb e http://www.botanica.org.br/acta/ojs 